\title{
Fractional variational approach with non-standard power-law degenerate Lagrangians and a generalized derivative operator
}

\author{
Rami Ahmad El-Nabulsi \\ College of Mathematics and Information Science, Neijiang Normal University, Neijiang, Sichuan 641112, China and \\ Athens Institute for Research and Education, Athens, Greece \\ E-mail: nabulsiahmadrami@yahoo.fr
}

\begin{abstract}
We extend the fractional actionlike variational approach where we substitute the standard Lagrangian by a non-standard power-law Lagrangian holding a generalized derivative operator. We focus on degenerate Lagrangians for the constructed fractional formalism where we show that non-linear oscillators with damping solutions may be obtained from degenerate nonstandard Lagrangians which are linear in velocities. We explore as well the case of $2^{\text {nd }}$-order derivatives non-standard Lagrangians and we study the case where Lagrangians are linear in accelerations where damping solutions are obtained as well. It was observed that these extensions give another possibility to obtain more fundamental aspects which may have interesting classical effects.
\end{abstract}

2010 Mathematics Subject Classification. 37N05. 49K22, 49K10

Keywords. fractional actionlike variational approach, non-standard degenerate Lagrangians, generalized derivative operator

\section{Introduction}

It is well-known that the calculus of variations is applied fruitfully in sciences and engineering as it is capable to describe the dynamical behavior of a given system by means of the EulerLagrange equation. Regrettably the standard approach is limited as it somewhat fails to describe nonconservative systems. A valid and mathematically solid novel or an extended approach is therefore required. One possible extension of the calculus of variations is based on the fractional calculus. In fact, in recent years, there has been lot of works dedicated to the fractional actionlike variational approach with fractional derivatives and fractional integrals where different forms of the fractional Euler-Lagrange equations were obtained depending on the type of fractional functional or fractional Lagrangian systems used [1, 3, 26, 27, 31, 39, 40. Most recent and broadest overviews of applications of the fractional calculus of variations are found in 34, 36. One successful version of the fractional calculus of variations is known as the fractional actionlike variational approach (FALVA) formulated in details in [26, 27]. An advantage of this approach is that dissipative dynamical systems are described appropriately.

Besides, there are already a series of arguments which make obvious that the notion of nonstandard Lagrangians (NSL) introduced by Arnold in his classic masterpiece book 2 play an important role in different domains of applied mathematics, e.g. nonlinear differential equations [7, 8, 9, 10, and dissipative dynamical systems [12, 13, 14, 15, 16, 17, 21, 37, 38, 41, 42, 44, as well as in theoretical physics [18, 19, 20, 22]. In general, NSL are characterized by a deformed kinetic 
term and a deformed potential function, yet the Euler-Lagrange equation that results from the standard calculus of variations lead to equations of motion that correspond to physically attractive nonlinear dynamical systems. In [17, we have introduced two different types of NSL: the socalled exponentially NSL and the power-law NSL. The resulting equations of motion are based on the standard calculus of variations and it was observed that both types exhibit interesting dynamical properties. More recently, in [44, the application of generalized fractional operators was applied to NSL and the consequential approach has proved to be useful to understand dissipative systems. More recently degenerate NSL approach was also applied to nonlinear oscillators 21 where it was observed that the theory describes correctly oscillators characterized by a positiondependent mass which represent an interesting class of problem in different field of sciences and engineering. However, as it is well-known there exist different types of oscillators derived usually from non-degenerate Lagrangians, e.g. oscillators characterized by time-dependent masses and timedependent frequencies depending on the dynamical problem under study (see 28] and references therein). These types of oscillators could not be derived from degenerate Lagrangians which in their turns fail to portray damping oscillators.

In this paper, we would like to show that by merging the FALVA and degenerate NSL which are linear in velocities (mainly the power-law NSL (NSPL)) for the case of $1^{\text {st }}$-order Lagrangians derivative and linear in accelerations for the case of $2^{\text {nd }}$-order Lagrangians derivative, damping oscillators may be obtained directly. In addition, we will show that exotic complexified oscillators represent one particular class of solution. However in our approach, the time-derivative operator is replaced by a new type of generalized derivative operator (GDO) motivated from non-standard dynamics. More precisely, it was observed that in the theory of NSL discussed in [17] the total derivative operator which is defined by $d / d t=\partial / \partial t+\dot{q} \partial / \partial q+\ddot{q} \partial / \partial \dot{q}$ occurs naturally in the modified Euler-Lagrange equations. We will show that the fractional approach constructed in this paper will give us the opportunity to acquire more fundamental aspects on the theory of nonlinear damping oscillators which may have motivating classical consequences in dissipative dynamical systems.

The paper is organized as follows: In Sec. 2, we introduce the FALVA with an NSPL and a GDO, then we construct the corresponding Lagrangian formalism; in Sec. $\mathbf{3}$ we extend the formalism for the case of higher-order derivatives NSPL; finally conclusions are given in Sec. 4. The paper is accompanied by a number of illustrations.

\section{FALVA with non-standard power-law degenerate Lagrangians and a GDO}

We start by introducing the following definition:

Definition 2.1. The generalized derivative operator (GDO) of $1^{\text {st }}$-order is given by:

$$
D_{\alpha}=\frac{d}{d t}+\alpha \dot{q} \frac{\partial}{\partial q}
$$

where $\alpha$ is a real parameter and $\alpha \neq-1$. Obviously we have $D_{0}=d / d t$.

It is worth noting that $D_{\alpha} q=(1+\alpha) \dot{q}, D_{\alpha} q^{n}=(1+\alpha) q^{n-1} \dot{q}, D_{\alpha} \dot{q}=\ddot{q} D_{\alpha} L^{1+\xi}=(1+\xi) L^{\xi} D_{\alpha} L$ and $d\left(D_{\alpha} q\right) / d t=(1+\alpha) \ddot{q}$. For $\alpha=-1$, the following relations hold as well: $D_{-1} q=0, D_{-1} q^{n}=$ $0, D_{-1} \dot{q}=\ddot{q} D_{-1} L^{1+\xi}=(1+\xi) L^{\xi} D_{-1} L, d\left(D_{-1} q\right) / d t=0$ and hence the problem looses its mathematical aspects.

Definition 2.2. If $L\left(D_{\alpha} q, q, t\right): \mathfrak{R}^{2 n+1} \rightarrow \mathfrak{R}$ be a sufficiently smooth differentiable function subject to the boundary conditions $q(0)=A$ and $q(\tau)=B$, then we define the extended fractional 
actionlike action functional defined on the space of $C_{A, B}$ of $C^{2}$ curves $q \in[0, t] \rightarrow \mathfrak{R}^{n}$ for the case of an NSPL by $S: C_{A, B} \rightarrow \mathfrak{R}$ :

$$
S=\frac{1}{\Gamma(\beta)} \int_{0}^{\tau} L^{1+\xi}\left(D_{\alpha} q, q, t\right)(\tau-t)^{\beta-1} d t,
$$

where $\xi \in \Gamma$ is the Euler gamma function, $\beta$ is a real or complex parameter, $\tau$ is the observer time and $t$ is the intrinsic time with $t \neq \tau$.

It is notable that the eventuality for the action $S$ to be complexified is not ruled out in our arguments. Examples of complexified action dynamics have already been studied in connection with the problem of complexified Hamiltonian and Lagrangian dynamics [35, 43 and they have motivating theoretical interest in both classical and quantum theories.

The problem is to find a function $q$ for which the functional (2) subject to the given boundary conditions has an extremum. It is notable that the assumption $q \in C^{1}[0, t]$ is sufficient in our arguments.

Remark 2.1. When $\beta=1$, equation (1) is reduced to the action functional for the case of a NSL introduced in [14] and when $\xi=0$, we find the action functional introduced in [26, 27], i.e. the FALVA approach.

Theorem 2.1 (Generalized Fractional Euler-Lagrange equations). If $q(t)$ is a solution to the problem of finding a function $q(t)$ that extremizes the fractional functional (2) subject to given boundary conditions, then the following generalized fractional Euler-Lagrange equation holds:

$$
\frac{\partial L\left(D_{\alpha} q, q, t\right)}{\partial q}-\frac{1}{L^{\xi}\left(D_{\alpha} q, q, t\right)} D_{\alpha}\left(L^{\xi}\left(D_{\alpha} q, q, t\right) \frac{\partial L\left(D_{\alpha} q, q, t\right)}{\partial D_{\alpha} q}\right)=\frac{\beta-1}{t-\tau} \frac{\partial L\left(D_{\alpha} q, q, t\right)}{\partial D_{\alpha} q} .
$$

The proof follows from [17, 26, 27, 32] after replacing $L \rightarrow L^{1+\xi}$ and $\frac{d}{d t} \rightarrow D_{\alpha}$.

Remark 2.2. When $\xi=0$, equation (3) is reduced to the fractional Euler-Lagrange equation derived in [26, 27] and when $\beta=1$, it is reduced to reduced to the modified Euler-Lagrange equation derived in 17 .

Corollary 2.1. The generalized Euler-Lagrange equation takes the $2^{\text {nd }}$ useful form:

$$
\begin{gathered}
=\frac{\partial L\left(D_{\alpha} q, q, t\right)}{\partial q}-\frac{d}{d t}\left(\frac{\partial L\left(D_{\alpha} q, q, t\right)}{\partial D_{\alpha} q}\right)-\alpha \dot{q} \frac{\partial}{\partial q}\left(\frac{\partial L\left(D_{\alpha} q, q, t\right)}{\partial D_{\alpha} q}\right)-\frac{\beta-1}{t-\tau} \frac{\partial L\left(D_{\alpha} q, q, t\right)}{\partial D_{\alpha} q} \\
=\frac{\xi}{L\left(D_{\alpha} q, q, t\right)}\left(\frac{\partial L\left(D_{\alpha} q, q, t\right)}{\partial t}+(1+\alpha) \dot{q} \frac{\partial L\left(D_{\alpha} q, q, t\right)}{\partial q}+(1+\alpha) \ddot{q} \frac{\partial L\left(D_{\alpha} q, q, t\right)}{\partial D_{\alpha} q}\right) \frac{\partial L\left(D_{\alpha} q, q, t\right)}{\partial D_{\alpha} q}
\end{gathered}
$$

Proof: We can write equation (3) as:

$$
\frac{\partial L\left(D_{\alpha} q, q, t\right)}{\partial q}-\frac{d}{d t}\left(\frac{\partial L\left(D_{\alpha} q, q, t\right)}{\partial D_{\alpha} q}\right)-\alpha \dot{q} \frac{\partial}{\partial q}\left(\frac{\partial L\left(D_{\alpha} q, q, t\right)}{\partial D_{\alpha} q}\right)-\frac{\beta-1}{t-\tau} \frac{\partial L\left(D_{\alpha} q, q, t\right)}{\partial D_{\alpha} q}
$$

Using $D_{\alpha} L^{1+\xi}\left(D_{\alpha} q, q, t\right)=(1+\xi) L^{\xi} D_{\alpha} L\left(D_{\alpha} q, q, t\right), d\left(D_{\alpha} q\right) / d t=(1+\alpha) \ddot{q}$ and the chain rule: 
we obtain equation

$$
\begin{gathered}
=\frac{\xi}{L\left(D_{\alpha} q, q, t\right)}\left(\frac{d}{d t}+\alpha \dot{q} \frac{\partial}{\partial q}\right) L\left(D_{\alpha} q, q, t\right) \frac{\partial L\left(D_{\alpha} q, q, t\right)}{\partial D_{\alpha} q} \\
\frac{d L\left(D_{\alpha} q, q, t\right)}{d t}=\frac{\partial L\left(D_{\alpha} q, q, t\right)}{\partial t}+\dot{q} \frac{\partial L\left(D_{\alpha} q, q, t\right)}{\partial q}+\frac{\partial L\left(D_{\alpha} q, q, t\right)}{\partial D_{\alpha} q} \frac{d\left(D_{\alpha} q\right)}{d t} \\
=\frac{\partial L\left(D_{\alpha} q, q, t\right)}{\partial t}+\dot{q} \frac{\partial L\left(D_{\alpha} q, q, t\right)}{\partial q}+(1+\alpha) \ddot{q} \frac{\partial L\left(D_{\alpha} q, q, t\right)}{\partial D_{\alpha} q}
\end{gathered}
$$

Remark 2.3. From equation (4), we observe the occurrence of another implicit GDO which is:

$$
\left(\frac{d}{d t}\right)_{\alpha} \equiv D_{\alpha}=\frac{\partial}{\partial t}+(1+\alpha) \dot{q} \frac{\partial}{\partial q}+\ddot{q} \frac{\partial}{\partial \dot{q}}
$$

and hence we can rewrite equation (4) as:

$$
\frac{\partial L\left(D_{\alpha} q, q, t\right)}{\partial q}-D_{\alpha}\left(\frac{\partial L\left(D_{\alpha} q, q, t\right)}{\partial D_{\alpha} q}\right)-\frac{\beta-1}{t-\tau} \frac{\partial L\left(D_{\alpha} q, q, t\right)}{\partial D_{\alpha} q}=\frac{\xi}{L\left(D_{\alpha} q, q, t\right)} D_{\alpha} L\left(D_{\alpha} q, q, t\right) \frac{\partial L\left(D_{\alpha} q, q, t\right)}{\partial D_{\alpha} q} .
$$

Illustrations:

To illustrate, we choose the degenerate time-independent NSL $L\left(D_{\alpha} q, q, t\right)=D_{\alpha} q+b q=(1+$ $\alpha) \dot{q}+b q$. Equation (4) gives straightforwardly the following $2^{\text {nd }}$-order linear differential equation of motion:

$$
2 \xi(1+\alpha) \ddot{q}+((2+\alpha) \xi-b(1+\alpha)) b \dot{q}+\frac{(\beta-1)(1+\alpha)}{t-\tau} \dot{q}+\frac{\beta-1}{t-\tau} b q-b^{2} q=0 .
$$

If we set for numerical purpose $\xi=-\frac{1}{2}, \alpha=\frac{1}{2}, b=-1, \beta=\frac{1}{2}$, then equation (5) is reduced to:

$$
6 \ddot{q}+\underbrace{\dot{q}-\frac{3}{t-\tau} \dot{q}}_{\text {dampingterms }}+\underbrace{\left(4-\frac{2}{t-\tau}\right)}_{\text {time-dependentfrequency }} q=0,
$$

and the solution is given by:

$$
q(T)=e^{-\frac{1}{12} i(\sqrt{95}-i) T} T^{\frac{3}{2}}\left(c_{1} U\left(\frac{5}{4}-\frac{7 i}{4 \sqrt{95}}, \frac{5}{2}, \frac{1}{6} i \sqrt{95} T\right)+c_{2} L_{-\frac{4}{3}+\frac{3}{4 \sqrt{95}}}^{\frac{3}{6}}\left(\frac{1}{6} i \sqrt{95} T\right)\right),
$$

where $c_{j}, j=1,2, \ldots$ are integration constants, $T=t-\tau, i=\sqrt{-1} \in C, U(a, b, x)$ is the confluent hypergeometric function of the $2^{\text {nd }}$ kind and $L_{n}^{a}(x)$ is the associated Laguerre polynomial. This solution describes harmonic oscillator with damping terms and a time-dependent frequency. However, for $\xi=-\frac{1}{2}, \alpha=1, b=-\frac{3}{4} \beta=\frac{1}{2}$, equation (5) is reduced to:

$$
32 \ddot{q}+\underbrace{\frac{16}{t-\tau} \dot{q}}_{\text {dampingterm }}+\underbrace{\left(9-\frac{6}{t-\tau}\right)}_{\text {time-dependentfrequency }} q=0,
$$


and the solution is given by:

$$
q(T)=e^{-\frac{3}{4 \sqrt{2}} i T} \sqrt{T}\left(c_{3} U\left(\frac{1}{8}(6-i \sqrt{2}), \frac{3}{2}, \frac{3 i T}{2 \sqrt{2}}\right)+c_{4} L_{\frac{1}{8}(6 i+\sqrt{2})}^{\frac{1}{2}}\left(\frac{3 i T}{2 \sqrt{2}}\right)\right),
$$

We plot respectively in Figures 1 and 2 sample individual solutions for (6) and (8) and for different initial conditions:
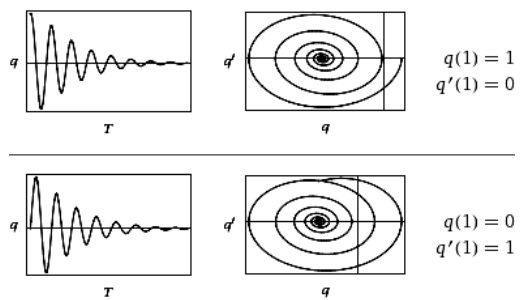

Fig. 1: Sample individual solution of equation (6)

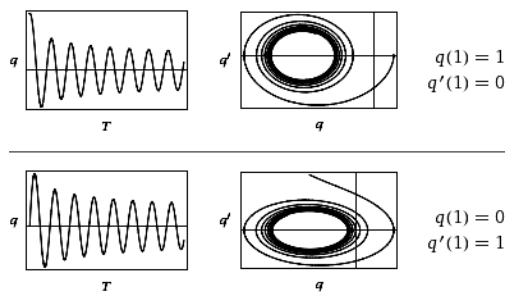

Fig 2: Sample individual solution of equation (8).

If we set $\beta=1$, we can have an idea about the main difference between the NSL approach constructed in [17] and FALVA with NSL+GDO. Hence, if we set $\xi=-\frac{1}{2}, \alpha=\frac{1}{2}, b=-1, \beta=1$, equation (5) is reduced to: $6 \ddot{q}+\dot{q}+4 q=0$ and the solution is simply given by

$$
q(T)=e^{-\frac{T}{12}}\left(c_{5} \sin \left(\frac{\sqrt{95} T}{12}\right)+c_{6} \cos \left(\frac{\sqrt{95} T}{12}\right)\right) .
$$

However, for $\xi=-\frac{1}{2}, \alpha=1, b=-\frac{3}{4} \beta=1$, equation (5) is reduced to $32 \ddot{q}+9 q=0$ and the solution is simply given by

$$
q(T)=c_{7} \sin \left(\frac{3 T}{4 \sqrt{2}}\right)+c_{8} \cos \left(\frac{3 T}{4 \sqrt{2}}\right) .
$$

We plot respectively in Figures 3 and 4 sample individual solutions for (10) and (11) and for different initial conditions: 


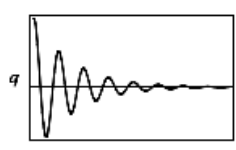

$T$

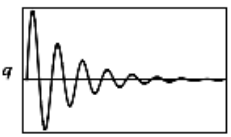

$T$

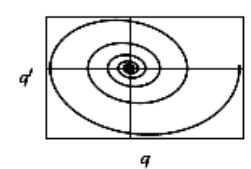

$q(0)=1$

$q^{\prime}(0)=0$

Fig. 3: Sample individual solution of equation (10)
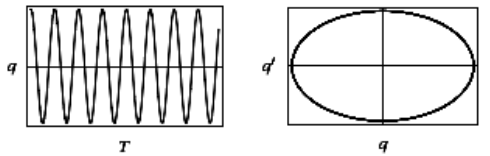

$q(0)=1$

$q^{\prime}(0)=0$
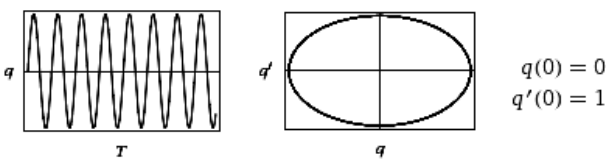

Fig. 4: Sample individual solution of equation (11)

Figures 1, 2 and 3 describe decaying dynamical systems with time due to the presence of dissipation, yet in Figure 4, we have standard oscillations yet the corresponding Lagrangian is non-standard. Nevertheless, the main difference between the NSL approach constructed in [17. and FALVA with NSL+GDO is that the case $\beta=1$ does not hold a time-dependent frequency and time-dependent damping terms. Besides, in the standard FALVA approach, oscillators with time-dependent frequencies are not obtained. It is noteworthy that all the previous approach fails for $\alpha=-1$, i.e. absence of the GDO.

We choose now the time-dependent NSL $L\left(D_{\alpha} q, q, t\right)=(t-\tau)\left(D_{\alpha} q+b q\right)$. Equation (4) results into the following equation of motion:

$$
\xi(1+\alpha) T \ddot{q}+(\xi-1)(1+\alpha) b T \dot{q}+(\xi+1)(1+\alpha) \dot{q}+(\xi+\beta) b q=0 .
$$

For $\xi=1, \alpha=\frac{1}{2}, \beta=\frac{1}{2}, b=1$, equation (12) is reduced to $T \ddot{q}+2 \dot{q}+q=0$ and the solution is given by:

$$
q(T)=\frac{1}{\sqrt{T}}\left(c_{9} J_{1}(2 \sqrt{T})-2 i c_{10} Y_{1}(2 \sqrt{T})\right)
$$

where $J_{n}(z)$ and $Y_{n}(z)$ are respectively the Bessel functions of $1^{\text {st }}$ and $2^{\text {nd }}$ kinds. However, if we choose the complexified NSL $L\left(D_{\alpha} q, q, t\right)=D_{\alpha} q+b(t-\tau) q$, then equation (4) gives:

$$
\xi(1+\alpha) \ddot{q}+\frac{\beta-1}{T}(1+\alpha) \dot{q}+(\xi-1)(1+\alpha) b T \dot{q}+(\xi+\beta-1) b q-b^{2} T^{2} q=0 .
$$


For $\xi=1, \beta=2, \alpha=-5, b=-2$, equation (14) is reduced to $\ddot{q}+\frac{1}{T} \dot{q}+q+T^{2} q=0$ which corresponds to a damping oscillator with a time-dependent frequency and the solution is given by:

$$
q(T)=\frac{e^{\frac{i T^{2}}{2} \sqrt{2 T^{2}}}}{T}\left(c_{11} U\left(\frac{1}{2}-\frac{i}{4}, 1,-i T^{2}\right)+c_{12} L_{-\frac{1}{2}+\frac{i}{4}}\left(-i T^{2}\right)\right) .
$$

We plot respectively in Figures 5 and 6 sample individual solutions for (13) and (15) and for different initial conditions:
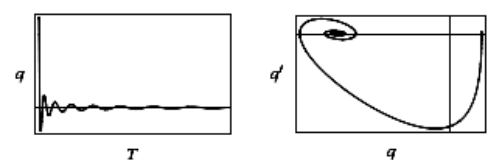

$q(1)=1$

$q^{\prime}(1)=0$
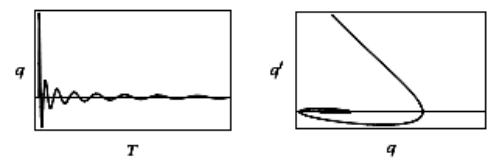

$q(1)=0$

$q^{\prime}(1)=1$

Fig. 5: Sample individual solution of equation (13)
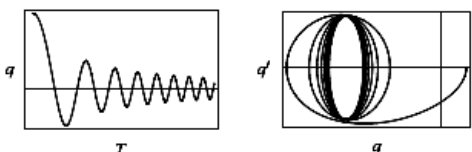

$q(1)=1$

$q^{\prime}(1)=0$
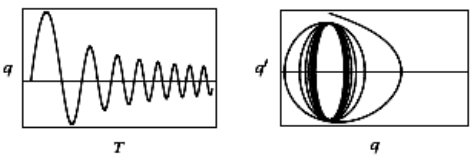

$q(1)=0$

$q^{\prime}(1)=1$

Fig. 6: Sample individual solution of equation (15)

It is notable that complexified oscillators or complex exotic oscillators which play an important role in quantum theory [11, 30, 33, 35, may also be obtained in our framework. To show this, we set in equation (14) $\xi=1, \alpha=1, \beta=2, b=-i$ which reduces equation (14) to

$2 \ddot{q}+\frac{2}{T} \dot{q}-2 i q+T^{2} q=0$ which corresponds to a damping oscillator with a complex

time-dependent frequency and having the following solution:

$$
q(T)=\frac{e^{\frac{i T^{2}}{2 \sqrt{2}} \sqrt{2 T^{2}}}}{T}\left(c_{13} U\left(\frac{2+\sqrt{2}}{4}, 1, \frac{i T^{2}}{\sqrt{2}}\right)+c_{14} L_{-\frac{2+\sqrt{2}}{4}}\left(\frac{i T^{2}}{\sqrt{2}}\right)\right) .
$$

We plot respectively in Figures 7 and 8 sample individual solutions for different initial conditions and a sample solution family in the complex plane for equation (16): 


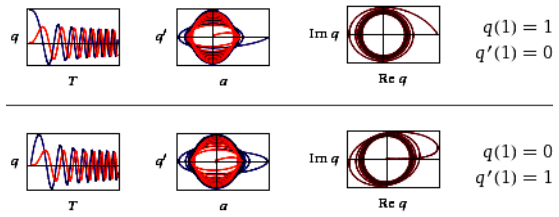

Fig. 7: Sample individual solution of equation (16)

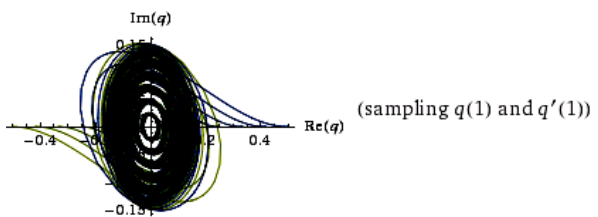

Fig. 8: Sample solution family of equation (16).

The blue line in Figure 3 corresponds for the real part, and the red line corresponds for the imaginary part. Under fixed initial conditions, these families of solutions show the oscillatory damping behavior of the system. Figure 4 shows symmetric closed and periodic complex trajectories in the complex $q$-plane representing the potential dynamics of the particle and enclosing real turning points. It is then obvious that real and complexified damping oscillators characterized by time-dependent masses and real and complexified time-dependent frequencies may be obtained directly from the present approach.

\section{FALVA with non-standard power-law Lagrangians and $2^{\text {nd }}$-order Lagrangian GDO}

In this section, we will extend the previous case for the case of FALVA with NSL holding $2^{\text {nd }}$-order GDO. It is notable that in the standard FALVA approach characterized by a standard Lagrangian with higher-order derivative [29, a Lagrangian holding a singular second-order derivative will not lead to any realistic equation of motion. This is also the case in the standard calculus of variations with second-order derivative. We will show in this section that both approaches may be solved by means of a GDO. We begin by introducing the following definition:

Definition 3.1: The generalized derivative operator (GDO) of $2^{\text {nd }}$-order is given by:

$$
D_{\alpha, \gamma}=\frac{d}{d t}+\alpha \dot{q} \frac{\partial}{\partial q}+\gamma \ddot{q} \frac{\partial}{\partial \dot{q}}
$$

where $\alpha$ and $\beta$ are real parameters.

The following relations hold accordingly: $D_{\alpha, \gamma} q=(1+\alpha) \dot{q}, D_{\alpha, \gamma} q^{n}=(1+\alpha) q^{n-1} \dot{q}$, $D_{\alpha, \gamma} \dot{q}=(1+\gamma) \ddot{q}$ and $d\left(D_{\alpha, \gamma} q\right) / d t=(1+\alpha) \ddot{q}$.

Definition 3.2. We define the extended fractional actionlike action functional for the case of an 
NSPL with $2^{\text {nd }}$ order Lagrangian derivative holding a $2^{\text {nd }}$-order GDO is defined by:

$$
S=\frac{1}{\Gamma(\beta)} \int_{0}^{\tau} L^{1+\xi}\left(D_{\alpha, \gamma} \dot{q}, D_{\alpha} q, q, t\right)(\tau-t)^{\beta-1} d t .
$$

Theorem 3.1 (Generalized $2^{\text {nd }}$-order Fractional Euler-Lagrange equations). The function $q=q(t)$ that extremizes the functional (18) necessarily satisfies the following modified Euler-Lagrange equations on $[0, \tau]$ :

$$
\begin{gathered}
\frac{\partial L\left(D_{\alpha, \gamma} \dot{q}, D_{\alpha} q, q, t\right)}{\partial q}-D_{\alpha}\left(\frac{\partial L\left(D_{\alpha, \gamma} \dot{q}, D_{\alpha} q, q, t\right)}{\partial D_{\alpha} q}\right)+D_{\alpha, \gamma} D_{\alpha, \gamma}\left(\frac{\partial L\left(D_{\alpha, \gamma} \dot{q}, D_{\alpha} q, q, t\right)}{\partial D_{\alpha, \gamma} \dot{q}}\right) \\
+\frac{\xi(\xi-1)}{L\left(D_{\alpha, \gamma} \dot{q}, D_{\alpha} q, q, t\right)} \frac{\partial L\left(D_{\alpha, \gamma} \dot{q}, D_{\alpha} q, q, t\right)}{\partial D_{\alpha, \gamma} \dot{q}}\left(D_{\alpha} L\left(D_{\alpha, \gamma} \dot{q}, D_{\alpha} q, q, t\right)\right)^{2} \\
+\frac{\xi}{L\left(D_{\alpha, \gamma} \dot{q}, D_{\alpha} q, q, t\right)} D_{\alpha} L\left(D_{\alpha, \gamma} \dot{q}, D_{\alpha} q, q, t\right) D_{\alpha}\left(\frac{\partial L\left(D_{\alpha, \gamma} \dot{q}, D_{\alpha} q, q, t\right)}{\partial D_{\alpha, \gamma} \dot{q}}\right)+\frac{(\beta-1)(\beta-2)}{(t-\tau)^{2}} \frac{\partial L\left(D_{\alpha, \gamma} \dot{q}, D_{\alpha} q, q, t\right)}{\partial D_{\alpha, \gamma} \dot{q}} \\
+\frac{\xi}{L\left(D_{\alpha, \gamma} \dot{q}, D_{\alpha} q, q, t\right)} \frac{\partial L\left(D_{\alpha, \gamma} \dot{q}, D_{\alpha} q, q, t\right)}{\partial D_{\alpha, \gamma} \dot{q}} D_{\alpha, \gamma} L\left(D_{\alpha, \gamma} \dot{q}, D_{\alpha} q, q, t\right)+\frac{1-\beta}{t-\tau} \frac{\partial L\left(D_{\alpha, \gamma} \dot{q}, D_{\alpha} q, q, t\right)}{\partial D_{\alpha} q} \\
-2 \frac{1-\beta}{t-\tau}\left(D_{\alpha}\left(\frac{\partial L\left(D_{\alpha, \gamma} \dot{q}, D_{\alpha} q, q, t\right)}{\partial D_{\alpha, \gamma} \dot{q}}\right)+\frac{\xi}{L\left(D_{\alpha, \gamma} \dot{q}, D_{\alpha} q, q, t\right)} D_{\alpha} L\left(D_{\alpha, \gamma} \dot{q}, D_{\alpha} q, q, t\right) \frac{\partial L\left(D_{\alpha, \gamma} \dot{q}, D_{\alpha} q, q, t\right)}{\partial D_{\alpha, \gamma} \dot{q}}\right) \\
+\frac{\xi}{L\left(D_{\alpha, \gamma} \dot{q}, D_{\alpha} q, q, t\right)} D_{\alpha} L\left(D_{\alpha, \gamma} \dot{q}, D_{\alpha} q, q, t\right)\left(D_{\alpha}\left(\frac{\partial L\left(D_{\alpha, \gamma} \dot{q}, D_{\alpha} q, q, t\right)}{\partial D_{\alpha, \gamma} \dot{q}}\right)-\frac{\partial L\left(D_{\alpha, \gamma} \dot{q}, D_{\alpha} q, q, t\right)}{\partial D_{\alpha, \gamma} \dot{q}}\right)=0 .
\end{gathered}
$$

The proof follows from [17, 26, 27, 32] after replacing $L \rightarrow L^{1+\xi}, \frac{d}{d t} \rightarrow D_{\alpha}$ and $\frac{d^{2}}{d t^{2}} \rightarrow D_{\alpha, \beta}$.

Remark 3.1. For $\alpha=\gamma=\xi=0$, equation (14) is reduced to the Euler-Lagrange equation obtained in the FALVA approach with $2^{\text {nd }}$-order derivatives:

$$
\begin{gathered}
\frac{\partial L(\ddot{q}, \dot{q}, q, t)}{\partial q}-\frac{d}{d t}\left(\frac{\partial L(\ddot{q}, \dot{q}, q, t)}{\partial \dot{q}}\right)+\frac{d^{2}}{d t^{2}}\left(\frac{\partial L(\ddot{q}, \dot{q}, q, t)}{\partial \ddot{q}}\right)+\frac{1-\beta}{t-\tau} \frac{\partial L(\ddot{q}, \dot{q}, q, t)}{\partial \dot{q}} \\
+\frac{(\beta-1)(\beta-2)}{(t-\tau)^{2}} \frac{\partial L(\ddot{q}, \dot{q}, q, t)}{\partial \ddot{q}}-2 \frac{1-\beta}{t-\tau} \frac{d}{d t}\left(\frac{\partial L(\ddot{q}, \dot{q}, q, t)}{\partial \ddot{q}}\right)=0 .
\end{gathered}
$$

\section{Illustrations:}

We choose the singular time-independent NSL

$L\left(D_{\alpha, \gamma} \dot{q}, D_{\alpha} q, q, t\right)=A D_{\alpha, \gamma} \dot{q}+C q=A(1+\gamma) \ddot{q}+C q,(A, C) \in$. Equation (19) gives $\xi=1$ for the following nonlinear $3^{r d}$-order differential equation of motion:

$$
\left(C+\frac{(\beta-1)(\beta-2) A}{(t-\tau)^{2}}\right)(A(1+\gamma) \ddot{q}+C q)-2 A \frac{1-\beta}{t-\tau}(A(1+\gamma) \dddot{q}+(1+\alpha) C \dot{q})=0
$$


If we set for illustration purpose $A=\frac{1}{2}, C=2$ and $\alpha=1, \beta=2, \gamma=1$ then equation (21) is reduced to:

$$
2 \ddot{q}+4 q+\frac{1}{t-\tau}(\dddot{q}+4 \dot{q})=0 .
$$

Whereas for $A=\frac{1}{2}, C=2$ and $\alpha=-\frac{1}{2}, \beta=2, \gamma=1$, equation (19) is reduced to:

$$
2 \ddot{q}+4 q+\frac{1}{t-\tau}(\dddot{q}+\dot{q})=0
$$

We solve equations (22) and (23) numerically and we plot their solutions for different initial conditions in Figures 9 and 10:
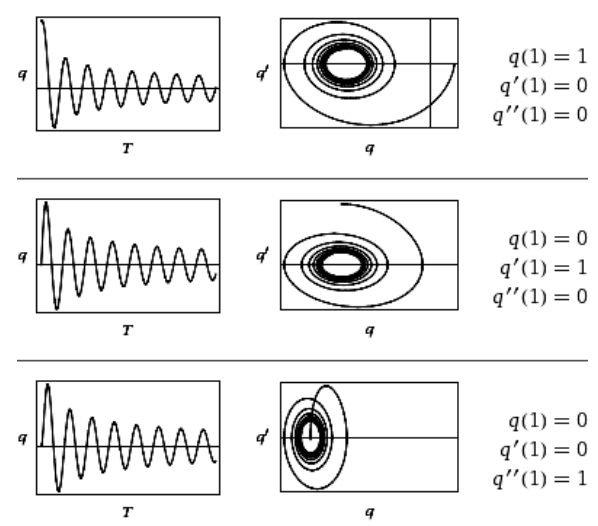

Fig.9: Sample individual solution of equation (22)

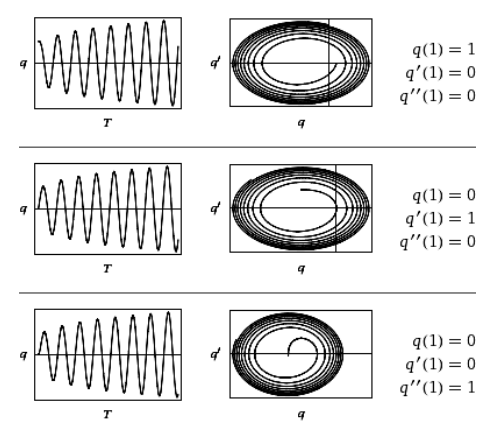

Fig. 10: Sample individual solution of equation (23)

We select now the time-dependent NSL $L\left(D_{\alpha, \gamma} \dot{q}, D_{\alpha} q, q, t\right)=(t-\tau)\left(D_{\alpha, \gamma} \dot{q}+C q\right)=(t-\tau)((1+$ $\gamma) \ddot{q}+C q), C \in$. Equation (19) gives:

$$
\begin{gathered}
C T+\frac{\xi(\xi-1)}{L} T\left(D_{\alpha} L\right)^{2}+\frac{\xi}{L} D_{\alpha} L+\frac{(\beta-1)(\beta-2)}{T^{2}} T \\
+\frac{\xi}{L} T D_{\alpha, \gamma} L-2 \frac{1-\beta}{T}\left(1+\frac{\xi T}{L} D_{\alpha} L\right)+\frac{\xi}{L} D_{\alpha} L(1-T)=0,
\end{gathered}
$$


where

$$
D_{\alpha} L=\frac{d L}{d t}+\alpha \dot{q} \frac{\partial L}{\partial q}=T(1+\gamma) \dddot{q}+(1+\gamma) \ddot{q}+(\alpha+1) C T \dot{q}+C q
$$

and

$$
D_{\alpha, \gamma} L=\frac{d L}{d t}+\alpha \dot{q} \frac{\partial L}{\partial q}+\gamma \ddot{q} \frac{\partial L}{\partial \dot{q}}=T(1+\gamma) \dddot{q}+(1+\gamma) \ddot{q}+(\alpha+1) C T \dot{q}+C q .
$$

For illustration purpose we set $\xi=1, \beta=2, C=1, \alpha=\frac{1}{4}, \gamma=1$ which reduces equation (24) to $C T L+4 D_{\alpha} L+2=0$ or more explicitly to:

$$
2 T \dddot{q}+2\left(T^{2}+4\right) \ddot{q}+T \dot{q}+T^{2} q+4 q+2=0 .
$$

For $\xi=1, \beta=1, C=1, \alpha=\frac{1}{4}, \gamma=1$, i.e. absence of the fractional formalism, equation (27) is reduced to:

$$
4 T \dddot{q}+2\left(1+T^{2}\right) \ddot{q}+2 T \dot{q}+T^{2} q+q=0 .
$$

We solve equations (27) and (28) numerically and we plot their solutions for different initial conditions in Figures 11 and 12:
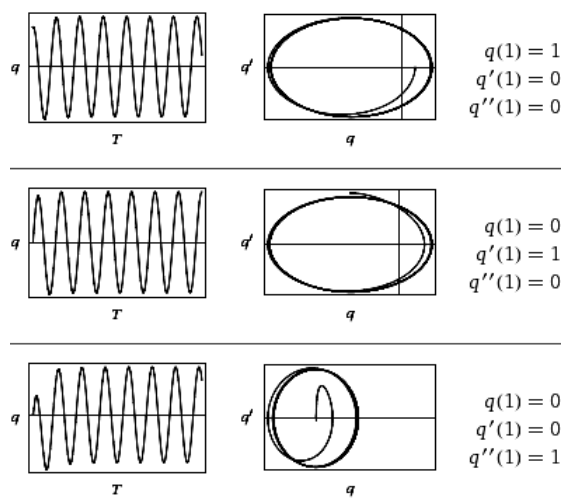

Fig. 11:Sample individual solution of equation (27)
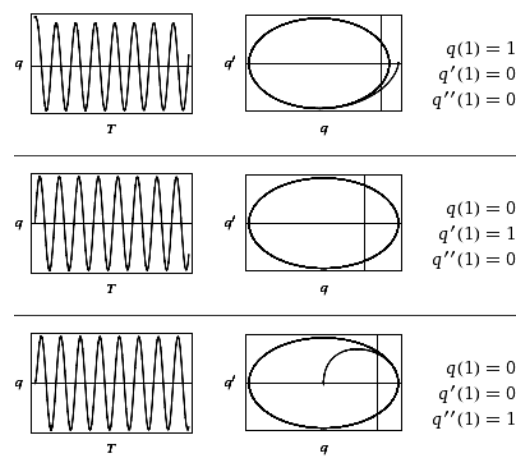

Fig. 12: Sample individual solution of equation (28) 
The dynamics differs slightly between both equations. It is obvious that an oscillatory behaviour is obtained whereas the dynamics is governed by $3^{r d}$-order differential equations. In reality, oscillatory solutions of $3^{r d}$-order differential equations were discussed in [4, 5, 6]] but through a different approach. Our approach is based on the presence of a generalized derivative operator in the Lagrangian formulation of FALVA whereas in [4, 5, 6, quasiderivatives vanishing at infinity were used.

Exotic complexified oscillators may also be obtained in our approach. By setting $\alpha=-1, C=$ $1+\gamma, 2 \beta=-i$, equation (27) will be reduced to the $3^{r d}$-order linear differential equation:

$$
T \dddot{q}+\ddot{q}+i T^{2} \ddot{q}+i T^{2} q+q=0 .
$$

Figures 13 and 14 illustrate sample individual numerical solutions for different initial conditions and a sample solution family in the complex plane for equation (29):

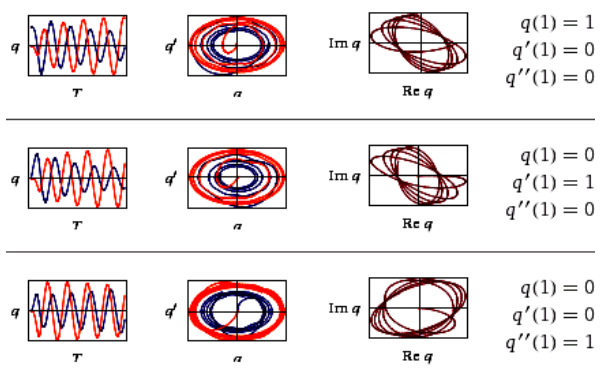

Fig. 13: Sample individual solution of equation (29)

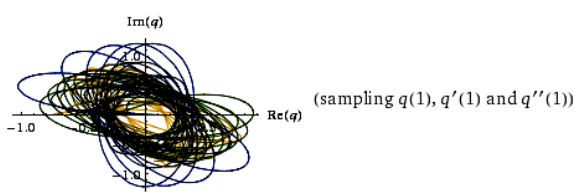

Fig.14: Sample solution family of equation (29)

Once more, these families of solutions demonstrate the oscillatory behavior of the dynamical system. These solutions are characterized by complexified Lagrangians which generalize the classical Lagrangian mechanics due to expansion of coordinates and momenta to an imaginary region. They are considered seriously in literature through different aspects (see [35, 43. and references therein).

All previous approaches may be extended for the case of higher-order GDO defined by:

$$
D_{\alpha_{1}, \alpha_{2}, \ldots, \alpha_{n}}=\frac{d}{d t}+\alpha_{1} \dot{q} \frac{\partial}{\partial q}+\alpha_{2} \ddot{q} \frac{\partial}{\partial \dot{q}}+\ldots+\alpha_{n} q^{(n)} \frac{\partial}{\partial q^{(n-1)}}=\frac{d}{d t}+\sum_{i=1}^{n} \alpha_{i} q^{(i)} \frac{\partial}{\partial q^{(i-1)}},
$$

where $q^{(n)}=\partial^{n} q / \partial t^{n}$ and so on. 


\section{Conclusions and perspectives}

The purpose of this paper was to generalize the fractional actionlike variational approach where the standard Lagrangian is replaced by its non-standard power-law counterpart and the timederivative operator is replaced by a generalized derivative operator. The main goal was to show that singular Lagrangians in velocities (for the case of $1^{\text {st }}$-order derivatives Lagrangians) and singular Lagrangians in accelerations (for the case of $2^{\text {nd }}$-order derivatives Lagrangians) may exhibit oscillatory dynamics characterized by special interesting properties, e.g. time-dependent masses, time-dependent frequencies, exotic complexified oscillators and so on. These features are absent in the standard FALVA and in the standard calculus of variations approach where singular Lagrangians will not lead to any dynamics. It will be interesting to construct the corresponding Hamiltonian formalism in a future work. Some complementary analysis in connection with quantum dynamics will be investigated in a future work.

\section{References}

[1] R. Almeida and D. F. M. Torres, Calculus of variations with fractional derivatives and fractional integrals, Appl. Math. Lett. 22 (2009), 1816-1820.

[2] V. I. Arnold, Mathematical Methods of Classical Mechanics, New York: Springer, 1978.

[3] D. Baleanu, New applications of fractional variational principles, Rep. Math. Phys. 61 (2008), 199-206.

[4] M. Bartusek, On oscillatory solutions of third order differential equations with quasiderivatives, Fourth Mississippi State Conference on Differential Equations and Computational Simulations, Electronic Journal of Differential Equations, Conference 03, 1999, pp 1-11.

[5] M. Bartusek and Z. Dosla, Oscillatory criteria for nonlinear third order differential equations with quasiderivatives, Diff. Eqs. Dyn. Syst. 3 (1995), 251-268.

[6] M. Bartusek and J. Osicka, Asymptotic behaviour of solutions of a third-order nonlinear differential equation, Nonlinear Anal. 34 (1998), 653-664.

[7] J. G. Carinena, M. F. Ranada and M. Santander, Lagrangian formalism for nonlinear secondorder Riccati Systems: one-dimensional integrability and two-dimensional superintegrability, J. Math. Phys. 46 (2005), 062703-062721.

[8] V. K. Chandrasekar, S. N. Pandey, M. Senthilvelan and M. Lakshmanan, Simple and unified approach to identify integrable nonlinear oscillators and systems, J. Math. Phys. 47 (2006), 023508-023545.

[9] V. K. Chandrasekar, M. Senthilvelan and M. Lakshmanan, On the Lagrangian and Hamiltonian description of the damped linear harmonic oscillator, Phys. Rev. E72 (2005), 066203-066211.

[10] V. K. Chandrasekar, M. Senthilvelan and M. Lakshmanan, A Nonlinear oscillator with unusual dynamical properties, in Proceedings of the Third National Systems and Dynamics, pp.1-4 (2006). 
[11] A. Déctor, H. A. Morales-Técotl, L. F. Urrutia and J. D. Vergara, An alternative canonical approach to the ghost problem in a complexified extension of the Pais-Uhlenbeck oscillator, SIGMA 5 (2009), 053, 22p.

[12] R. A. El-Nabulsi, Non-standard fractional Lagrangians, Nonlinear Dyn. 74 (2013), 381-394.

[13] R. A. El-Nabulsi, Non-standard Lagrangians in rotational dynamics and the modified NavierStokes equation, Nonlinear Dyn. 79 (2015), 2055-2068.

[14] R. A. El-Nabulsi, Fractional oscillators from non-standard Lagrangians and time-dependent fractional exponent, Comp. Appl. Math. 33 (2014), 163-179.

[15] R. A. El-Nabulsi, Non-standard non-local-in-time Lagrangian in classical mechanics, Qual. Theory Dyn. Syst. 13 (2014), 149-160.

[16] R. A. El-Nabulsi, T. Soulati and H. Rezazadeh, Non-standard complex Lagrangian dynamics, J. Adv. Res. Dyn. Contr. Theor. 5, No. 1 (2012), 50-62.

[17] R. A. El-Nabulsi, Nonlinear dynamics with non-standard Lagrangians, Qual. Theory Dyn. Syst. 13(2013), 273-291.

[18] R. A. El-Nabulsi, Modified Proca equation and modified dispersion relation from a power-law Lagrangian functional, Indian J. Phys. 87, No.5 (2013), 465-470; Erratum Indian J. Phys. 87, 10, (2013) p. 1059.

[19] R. A. El-Nabulsi, Quantum field theory from an exponential action functional, Indian J. Phys. 87, No. 4 (2013), 379-383.

[20] R. A. El-Nabulsi, Generalizations of the Klein-Gordon and the Dirac Equations from nonstandard Lagrangians, Proc. Nat. Acad. Sci. India Sec. A: Phys. Sci. 83 (2013), 383-387.

[21] R. A. El-Nabulsi, A generalized nonlinear oscillator from non-standard degenerate Lagrangians and its consequent Hamiltonian formalism, Proc. Nat. Acad. Sci. India Sec. A: Phys. Sci 84(4) (2014), 563-569.

[22] R. A. El-Nabulsi, Electrodynamics of relativistic particles through non-standard Lagrangian, J. At. Mol. Sci. 5(3) (2014), 268-279.

[23] R. A. El-Nabulsi, Non-standard power-law Lagrangians in classical and quantum dynamics, Appl. Math. Lett. 43 (2015), 120-127.

[24] R. A. El-Nabulsi, Classical string field mechanics with non-standard Lagrangians, Math. Sci. 9 (2015), 173-179.

[25] R. A. El-Nabulsi, From classical to discrete gravity through exponential non-standard Lagrangians in general relativity, Mathematics 3(3) (2015), 727-745.

[26] R. A. El-Nabulsi and D. F. M. Torres, Fractional actionlike variational problems, J. Math. Phys. 49 (2008), 053521-053529. 
[27] R. A. El-Nabulsi, A fractional approach of nonconservative Lagrangian dynamics, Fiz. A14, No. 4 (2005), 289-298.

[28] R. A. El-Nabulsi, The fractional calculus of variations from extended Erdelyi-Kober operator, Int. J. Mod. Phys. B23 (2009), 3349-3361.

[29] R. A. El-Nabulsi, Higher-order fractional field equations in $(0+1)$ dimensions and physics beyond the standard model, Fiz. A19 (2010), 55-72.

[30] R. A. El-Nabulsi, Lagrangian and Hamiltonian dynamics with imaginary time, J. Appl. Anal. 18(2) (2012), 283-295.

[31] G. S. Frederico and D. F. M. Torres, Necessary optimality conditions for fractional action-like problems with intrinsic and observer times, WSEAS Trans. Math. 7(1) (2008), 6-11.

[32] I. M. Gelfand, S. V. Fomin and R. A. Silverman, Calculus of variations, Englewood Cliffs, N.J.: Prentice-Hall Inc., 1963.

[33] S. Ghosh and S. K. Modak, Classical oscillator with position-dependent mass in a complex domain, Phys. Lett. A373 (2009), 1212-1217.

[34] R. Herrmann, Fractional Calculus: An Introduction for Physicists, World Scientific Publishing Company, 2011.

[35] R. S. Kaushal, Classical and quantum mechanics of complex Hamiltonian systems: An extended complex phase space approach, PRAMANA J. Phys. 73(2) (2009), 287-297.

[36] A. B. Malinowska and D. F. M. Torres, Introduction to the Fractional Calculus of Variations, Imperial College Press, London, UK, 2012.

[37] Z. E. Musielak, Standard and non-standard Lagrangians for dissipative dynamical systems with variable coefficients, J. Phys. A: Math. Theor. 41 (2008), 055205-055222.

[38] Z. E. Musielak, General conditions for the existence of non-standard Lagrangians for dissipative dynamical systems, Chaos Solitons and Fractals 42, No. 15 (2009), 2645-2652.

[39] T. Odzijewicz, A. B. Malinowska and D. F. M. Torres, Fractional calculus of variations in terms of a generalized fractional integral with applications to physics, Abs. Appl. Anal. 2012 (2012), Article ID 871912.

[40] T. Odzijewicz, A. B. Malinowska and D. F. M. Torres, A generalized fractional calculus of variations, Control and Cybernetics 42(2) (2013), 443-458.

[41] A. Saha and B. Talukdar, On the non-standard Lagrangian equations, arXiv: 1301.2667.

[42] A. Saha and B. Talukdar, Inverse variational problem for non-standard Lagrangians, Rep. Math. Phys. 73, (2014) 299-309.

[43] V. I. Sbitnev, Bohmian trajectories and the path integral paradigm. Complexified Lagrangian mechanics, Int. J. Bifur. Chaos 19(9) (2009), 2335-2346.

[44] G. S. Taverna and D. F. M. Torres, Generalized fractional operators for nonstandard Lagrangians, Math. Meth. Appl. Sci. 38 (2015), 1808-1812. 